\title{
Introduction to Fourth Special Issue on Electroporation-Based Technologies and Treatments
}

\author{
Damijan Miklavčič • Lluis M. Mir • \\ P. Thomas Vernier
}

Published online: 31 October 2014

(c) Springer Science+Business Media New York 2014

This fourth special electroporation-based technologies and treatments issue of the Journal of Membrane Biology contains reports on recent developments in the field of electroporation by participants in the 7th International Workshop and Postgraduate Course on electroporationbased technologies and treatments (EBTT 2013) held in Ljubljana, November 17-23, 2013. The 65 participants included faculty members, invited lecturers, special guests, and young scientists, and students from 16 countries. In addition to lectures on the fundamentals, this year's sessions included talks on microbial inactivation by pulsed electric fields, modeling of intracellular electroporation, electroporation in food processing, and electrotransfer-facilitated DNA vaccination. One of the strengths and attractions of this workshop-course is the practical training exercises offered each afternoon. Organized and led by colleagues from the Laboratory of Biocybernetics of the Faculty of Electrical Engineering at the University of Ljubljana and by visiting researchers, these afternoon laboratory sessions provide a unique opportunity for hands-on experience guided by experts,

\section{Miklavčič}

Faculty of Electrical Engineering, University of Ljubljana, Tržaška 25, 1000 Ljubljana, Slovenia

e-mail: damijan.miklavcic@fe.uni-lj.si

\section{M. Mir}

UMR 8203 Vectorology and Anti-cancerous Therapeutics, Institute Gustave-Roussy, 39 Rue Camille Desmoulins, 94805 Villejuif, France

e-mail: luismir@igr.fr

P. T. Vernier $(\bowtie)$

Frank Reidy Research Center for Bioelectrics, Old Dominion University, Norfolk, VA 23508, USA

e-mail: pvernier@odu.edu with activities ranging from fluorescence microscopy to electrical characterization of artificial membranes to molecular dynamics simulations. The school is now an established and unique platform for the acquisition of theoretical and practical knowledge of electroporation mechanisms and applications.

The workshop and postgraduate course was conducted within the scope of the European Associated Laboratory on Pulsed Electric Fields Applications in Biology and Medicine (LEA EBAM) and co-organized by COST TD1104 Action (http://www.electroporation.net/). COST-European Cooperation in Science and Technology is the oldest intergovernmental framework aimed at facilitating the collaboration and networking of scientists and researchers at the European level established in 1971 (http://www.cost.eu).

The peer-reviewed selection of articles in this issue provides a cross-section of the ongoing electroporationrelated research being carried out by participants at the Ljubljana meeting. We are grateful to the contributors for their efforts in presenting their recent results, which will challenge readers, whether new or old in the field, to evaluate assumptions and consider new ideas, and we are especially grateful to our scientific colleagues who reviewed the manuscripts.

Finally we acknowledge the indispensable support of the agencies, societies, and companies who sponsor the school, in particular the Slovenian Research Agency, the Centre National de la Recherche Scientifique (CNRS), and the Bioelectrochemical Society, which has sponsored the school from its very beginning, and also IGEA (Italy), Mikro + Polo (Slovenia), Iskra Medical (Slovenia), C3 M (Slovenia), BIA Separations (Slovenia), LTFE (Slovenia), Profector (Ireland), and the University of Bielefeld (Germany), who made it possible to increase student participation through reduced fees and lodging expenses. 
COST-European Cooperation in Science and Technology is an intergovernmental framework aimed at facilitating the collaboration and networking of scientists and researchers at European level. It was established in 1971 by 19 member countries and currently includes 35 member countries across Europe, and Israel as a cooperating state.

COST funds pan-European, bottom-up networks of scientists and researchers across all science and technology fields. These networks, called 'COST Actions', promote international coordination of nationally-funded research.

By fostering the networking of researchers at an international level, COST enables break-through scientific developments leading to new concepts and products, thereby contributing to strengthening Europe's research and innovation capacities.

COST's mission focuses in particular on:

- Building capacity by connecting high quality scientific communities throughout Europe and worldwide.

- Providing networking opportunities for early career investigators.

- Increasing the impact of research on policy makers, regulatory bodies and national decision makers as well as the private sector.

Through its inclusiveness, COST supports the integration of research communities, leverages national research investments, and addresses issues of global relevance.
Every year thousands of European scientists benefit from being involved in COST Actions, allowing the pooling of national research funding to achieve common goals.

As a precursor of advanced multidisciplinary research, COST anticipates and complements the activities of EU Framework Programmes, constituting a "bridge" toward the scientific communities of emerging countries. In particular, COST actions are also open to participation by non-European scientists coming from neighbor countries (for example Albania, Algeria, Armenia, Azerbaijan, Belarus, Egypt, Georgia, Jordan, Lebanon, Libya, Moldova, Montenegro, Morocco, the Palestinian Authority, Russia, Syria, Tunisia, and Ukraine) and from a number of international partner countries.

COST's budget for networking activities has traditionally been provided by successive EU RTD Framework Programmes. COST is currently executed by the European Science Foundation (ESF) through the COST Office on a mandate by the European Commission, and the framework is governed by a Committee of Senior Officials (CSO) representing all its 35 member countries.

More information about COST is available at www.cost. eu. 